\title{
Desigualdades sociais no Município do Rio de Janeiro: uma comparação entre os censos 1991 e 1996
}

\author{
Social inequalities in the city of Rio de Janeiro: \\ a comparison of the 1991 and 1996 censuses
}

\begin{abstract}
Alberto Lopes Najar 1
Luís Otávio Farias 1

Eduardo César Marques 2,3

Christina Zackiewicz 1
\end{abstract}

\footnotetext{
1 Departamento de Ciências Sociais, Escola Nacional de Saúde Pública, Fundação Oswaldo Cruz. Rua Leopoldo Bulhões 1480, Rio de Janeiro, $R J$ 21041-210, Brasil. najar@ensp.fiocruz.br faria@ensp.fiocruz.br zackz@sti.com.br

2 Departamento de Ciência Política, Universidade de São Paulo. Av. Prof. Luciano Gualberto 315 , São Paulo, SP 05508-900, Brasil. ecmarq@uol.com.br 3 Centro Brasileiro de Análise e Planejamento. Rua Morgado de Mateus 615 São Paulo, SP

04015-902, Brasil.
}

\begin{abstract}
This article presents a comparative model for the results of the 1996 and 1991 censuses, based on the relations indicated by the results from 1991. The authors conduct a reclassification of the social quality index (SQI) proposed by Najar (1997) for comparison of the two censuses, controlling for the variable "heads of households with 15 or more years of schooling". As is known, the 1996 census was conducted with the purpose of counting the population, and its scope of variables was quite limited. In this sense, the comparison presented here is exploratory and is limited to the variable that registers level of schooling for heads of private, permanent households, seeking to identify some changes. After situating the discussion from the perspective of analytical traditions on which it is based, the authors present and discuss the results, indicating important changes in the way the literature classically interpreted the distribution of social structure in Greater Metropolitan Rio de Janeiro. An increase was observed in years of schooling in the Rio de Janeiro population, especially among female heads of households. From the perspective of methodological tradition, this study is also in keeping with the groundbreaking work of Vetter (1981) and Pinçon-Charlot et al. (1986).
\end{abstract}

Key words Social Inequity; Demographic Analysis; Censuses

Resumo O artigo apresenta um modelo de comparação entre os resultados dos censos de 1996 e de 1991, a partir das relações indicadas pelos resultados deste último. Fez-se uma reclassificação do Índice de Qualidade Social (IQS) proposto em Najar (1997) para a comparação dos dois censos, controlando-se para a variável "chefes de domicílio com 15 ou mais anos de estudo". Como se sabe, o censo 1996 foi realizado com a finalidade da contagem de população, sendo seu âmbito de variáveis bastante limitado. Nesse sentido, a comparação que se apresenta tem caráter exploratório e se limitará à variável que registrou a escolaridade para os chefes dos domicílios particulares e permanentes, procurando identificar algumas mudanças. Após situar a discussão do ponto de vista das tradições analíticas em que se apóia, apresentam-se e discutem-se os resultados que indicam importantes transformações na maneira como classicamente a literatura interpretou a distribuição da estrutura social no espaço metropolitano carioca. Verificou-se a elevação, em termos de anos de escolaridade, da população carioca, em especial para o segmento de mulheres chefes de domicílio. Este trabalho alinha-se, do ponto de vista da tradição metodológica, com os importantes trabalhos de Vetter (1981) e Pinçon-Charlot et al. (1986).

Palavras-chave Iniqüidade Social; Análise Demográfica; Censos 


\section{Introdução}

Nos anos 1970, produziram-se no Rio de Janeiro diversos trabalhos sobre a estruturação intra-urbana, consolidando-se um modo de entender as metrópoles brasileiras que exerceriam forte influência sobre os estudos e pesquisas posteriores em todo o Brasil. Merece destaque, nesse sentido, o trabalho de autoria do Instituto de Pesquisa Econômica Aplicada e do Instituto Brasileiro de Administração Municipal (IPEA/IBAM, 1976), que consolidou extensa pesquisa realizada no IBAM. Esse estudo, de caráter eminentemente operacional, associava-se aos esforços para a produção de mecanismos técnicos de gestão dos aglomerados metropolitanos em todo o Brasil, criados pela Lei Complementar 14/1974, e, mais especificamente, para as áreas da região metropolitana do Rio de Janeiro, incorporadas a um único poder estadual com a fusão dos Estados do Rio de Janeiro e da Guanabara em julho de 1974 (Lei Complementar 20/1974). Ele representou um marco inovador nas análises sobre aglomerados metropolitanos, pois, pela primeira vez, o aglomerado metropolitano do Rio de Janeiro e o seu entorno foram descritos e analisados como uma estrutura em que um núcleo metropolitano forte e hipertrofiado, concentrador de serviços e de recursos econômicos, articulavase a uma vasta periferia, organizada de forma concêntrica em gradiente decrescente de recursos e de integração ao núcleo. Compor-seia, então, a região metropolitana do Rio de Janeiro de quatro anéis: o núcleo, as periferias imediata, intermediária e distante.

O trabalho que divulgou este modelo de forma ampla (Santos \& Bronstein, 1978), investigava a relação entre o modelo de desenvolvimento adotado pós-1964 e a organização dos espaços metropolitanos, concluindo que o Rio de Janeiro "está servindo e servirá cada vez mais de modelo de metropolização para as cidades brasileiras. Em outros termos, o Rio tende a ditar a moda metropolitana" (Santos \& Bronstein, 1978:7), vale dizer a tendência de desenvolver núcleos hipertrofiados cercados de periferias crescentemente carentes de serviços e infra-estrutura, habitados por população de baixa renda. Segundo essa formulação, o Rio de Janeiro e outras cidades brasileiras se estruturariam de forma oposta às cidades americanas. Os ricos, nas cidades brasileiras, amontoam-se próximos da infra-estrutura existente no centro da metrópole; os pobres, por sua vez, habitam cidades-dormitório distantes e carentes. Esse modelo, talvez por sua simplicidade e capacidade operacional, exerceu e continua exercendo, influência sobre estudos dos mais variados matizes, do excelente trabalho de Abreu (1987) sobre a construção histórica do espaço carioca aos trabalhos na área da epidemiologia, como os de Duchiade (1991) e Leal (1995), e na de planejamento em saúde, como os de Vasconcellos (1997) e Galvão et al. (1999).

O modelo núcleo-periferias concêntricas para o Rio de Janeiro tinha inicialmente objetivos meramente instrumentais e de descrição geral, e, nesse contexto, poderia ser considerado apropriado. A sua utilização posterior, no entanto, ultrapassou em muito aqueles objetivos, tornando-o problemático. Inicialmente porque a aplicação do modelo levou a certo determinismo das estruturas sobre o espaço. Isso fica claro, por exemplo, quando Santos (1980:22) afirmou que "podem ser verificadas, nas grandes cidades brasileiras, as formas de estruturação espacial correspondentes à implantação e ao desenvolvimento do capitalismo no país". Excessivamente genérica, a afirmação ao mesmo tempo em que diz tudo, não diz nada, pois sem dúvida as relações sociais se inscrevem concretamente nos espaços, mas como muito bem afirmou Massey (1992:70), “in one way or another, geography matters". Se assim não fosse, todos os países do terceiro mundo teriam seus espaços construídos de forma semelhante, e isso não ocorre porque o espaço herdado constrange os atores sociais em suas práticas e relações. O espaço, assim como acontece com as instituições, produz ao mesmo tempo em que é produzido pela sociedade. Esta dialética é intrínseca, visto que o espaço é uma das dimensões da vida e portanto, da sociedade, o que torna impossível descobrir quem produz ou reproduz quem.

O segundo problema de utilização do modelo é de natureza metodológica: excessivamente simplificador da complexidade metropolitana, levou boa parte das análises a tratar como similares espaços dessemelhantes sob o ponto de vista de sua composição e constituição social. Já no início da década, Vetter (1981: 596), advertia que "talvez seja interessante passar a pensar em termos de um sistema de núcleos e periferias, uma vez que a estrutura interna da cidade parece muito mais variada do que poderia ser explicada por uma simples estrutura núcleo-periferia". Marques (1993) pôde comprovar a existência, já em 1980, de conteúdos sociais diversos em espaços considerados homogêneos pela literatura. A estruturação interna da região metropolitana do Rio de Janeiro era aparentemente não concêntrica e não contígua entre espaços com conteúdos sociais similares, assemelhando-se muito mais a um mo- 
saico complexo do que a um gradiente decrescente de círculos concêntricos. Foi exatamente esse aspecto que se revelou em outras análises (Najar, 1997, 1998; Najar et al., 2000): o aglomerado metropolitano comandado pela cidade do Rio de Janeiro se apresentou, não como uma cidade segregada, partida (Ribeiro \& Telles, 2000; Ventura, 1994), mas como uma heterópolis, no sentido empregado por Jencks (1993), um verdadeiro e sofisticado mosaico urbano (Timms, 1971), marcado tanto por dégradés quanto por dicotomias, desautorizando-nos a pensar o espaço urbano carioca como uma cidade dual, visto que ele se nos apresentava como uma cerzidura, um mosaico complexo, bem mais próximo ao que parece ter-se transformado a própria sociedade brasileira nas últimas décadas. Alguns trabalhos recentes têm indicado que, no interior deste, apesar da melhoria das condições gerais de vida ao longo dos anos 1980, persistem lugares em condições precárias onde predomina uma superposição de carências (Torres \& Marques, 2001).

No sentido de explorar esse aspecto de mosaico da estrutura sócio-espacial da cidade do Rio de Janeiro, a abordagem apresentada apóiase na construção de uma classificação hierárquica crescente a partir de uma análise de componentes principais, seguida de um estudo de aglomeração (cluster), apresentada em detalhes, para toda a região metropolitana do Rio de Janeiro, em Najar et al. (2000), ou de um ponto de vista mais teórico, em Pinçon-Charlot et al. (1986); Dunteman (1989); Rummel (1970); Hamilton (1992); e Johnson \& Wichern (1992).

A construção do modelo analítico partiu de um cruzamento de indicadores sociais, de forma a que se pudesse analisar a distribuição das variáveis, agrupadas a partir dos seguintes blocos temáticos: demografia, renda, educação, infra-estrutura e serviços, condições da habitação/domicílio. Uma caracterização mais abrangente foi possível graças à utilização de técnicas de análise multivariada, opção também escolhida por diversos trabalhos recentes, especialmente Preteceille \& Valladares (2000), Marques (2000). O trabalho estabeleceu sete grupos de unidades, cujo resultado final foi um índice sintético (Índice de Qualidade Social IQS), que permitiu classificar as unidades de agregação da informação utilizadas segundo uma escala de categorias que, respeitados os seus limites, pode ser usada como proxy da qualidade social do território em exame.

O resultado da classificação acabou por indicar importantes transformações na forma como classicamente a literatura interpretou a distribuição da estrutura social no espaço me- tropolitano carioca. Os modelos utilizados consideram uma distribuição concêntrica, contínua e contígua entre espaços de características sociais similares a partir do centro da metrópole. Indicou a existência de um tecido social muito mais complexo na metrópole, nela espaços de características similares se encontram em locais distintos e não contíguos e os eixos de transportes (e seus custos) claramente não são o único, e nem o mais importante fator na localização das atividades e grupos no espaço. Embora as causas do padrão observado não tenham sido discutidas, é razoável sustentar a hipótese de que fatores ligados à história da cidade, à inércia dos diversos momentos da estruturação do espaço, a estratégias, interesses e valores de cada grupo social (referentes a cada espaço) e à cultura urbana conformem o núcleo causal da estruturação do espaço metropolitano.

Ao se investigar a diferenciação sócio-espacial na região, os resultados da análise confirmaram a constatação de diversos trabalhos que indicam, por exemplo, que há bairros e favelas dentro de uma mesma favela (Machadoda-Silva, 1967; Marques, 1993; Preteceille \& Valladares, 2000; Vetter, 1981). Especialmente com relação às favelas cariocas, os resultados a que chegamos, assim como outros tantos trabalhos (Preteceille \& Valladares, 2000; Valladares, 2001, em apresentação feita durante o Fórum América Latina Habitar 2000), recomendam que se deve ter cuidado, por exemplo, ao se discutir a periferização das favelas em relação à metrópole.

É nesse alinhamento analítico que se enquadram as hipóteses e suposições feitas neste artigo, em que se compararão o censo 1996 (IBGE, 1996) com os resultados do censo 1991 (IBGE, 1991), a partir das relações indicadas pelos resultados deste último, ou seja, se empregará uma reclassificação do IQS a fim de que se compararem os dois censos.

\section{Material e métodos}

A análise de componentes principais (ACP) é uma técnica estatística que transforma um conjunto de variáveis em um conjunto menor e não-correlacionado, composto por fatores que maximizam a informação do conjunto original de variáveis. Um conjunto menor de variáveis é, portanto, mais fácil de compreender e manipular do que uma matriz analítica extensa. Como resultado pode-se lidar com os dados de maneira mais parcimoniosa, o que possibilita uma compreensão mais aperfeiçoa- 
da sobre a estrutura dos dados. Em função da exigüidade de espaço para apresentar em detalhe a referida técnica, remete-se os leitores interessados para Rummel (1970). As correlações observadas entre as variáveis resultam da partilha destes fatores, ou dimensões comuns. Os fatores são estimados como combinações lineares das variáveis originais. Os resultados das análises apresentadas em Najar (1997, 1998), Najar et al. (2000) e Najar \& Marques (1998), permitem propor o IQS. Essa é, de fato, uma escala de classificação dos setores censitários da região metropolitana do Rio de Janeiro com as seguintes categorias: grupo I (IQS muito alto), grupo II (IQS alto), grupo III (IQS médio), grupo IV (IQS médio/baixo), grupo V (IQS baixo), grupo VI (IQS baixo/muito baixo) e grupo VII (IQS muito baixo).

A Tabela 1 a seguir, sintetiza os resultados para o censo 1991. Deve-se observar inicialmente, que apenas o primeiro fator (F1), expli-

Tabela 1

Síntese dos resultados da análise de componentes principais. Município do Rio de Janeiro, Brasil, 1991.

\begin{tabular}{lcc}
\hline Fator & \% da variância & \% acumulado \\
\hline 1 & 47.6 & 47.6 \\
2 & 19.4 & 67.1 \\
3 & 11.2 & 78.2 \\
4 & 6.2 & 84.5 \\
5 & 5.9 & 90.3 \\
6 & 4.5 & 94.8 \\
7 & 2.6 & 97.4 \\
\hline
\end{tabular}

ca $47,6 \%$ da variância. O modelo utilizado empregou apenas os dois primeiros fatores (F1 e F2), explicando-se com eles $67,1 \%$ da variância dos elementos envolvidos (Tabela 1). Esses dois fatores, por sua vez, apresentam uma relação quadrática entre si, o chamado efeito Gutman (Rummel, 1970), possibilitando uma solução a um fator, ainda mais parcimoniosa.

A observação da tabela Matriz de Fatores (Tabela 2), indica que as variáveis para as quais F1 apresenta coeficientes de relação mais altos são: (a) proporção de chefes de domicílio com 15 ou mais anos de estudo $(0,85)$, (b) rendimento nominal dos chefes de domicílio em salários-mínimos $(0,79)$ e (c) número de banheiros por domicílio $(0,74)$. Dessas variáveis, apenas a primeira é comum aos censos 1991 e 1996, tendo sido empregado um artifício metodológico para investigar o efeito do valor da variável escolaridade alta de 1996 em 1991: substituiu-se a proporção de chefes de domicílio com 15 ou mais anos de estudo, computada em 1991, pela mesma variável computada em 1996, repetiuse então os mesmos procedimentos de análise de componentes principais, seguidos de cluster, investigando-se o efeito dessa alteração. Em outras palavras, o valor da variável em 1991 assumiu o valor de 1996, ceteris paribus, tendo sido gerado novo índice de qualidade social que se chamou IQS91* e que foi comparado com o anterior, IQS91. A análise foi feita setor censitário a setor censitário (isso significa o exame de áreas que agregam entre 300 a 400 domicílios) tendo-se por referência os setores em 1991, o que acarretou a exclusão daqueles que foram desmembrados e/ou criados à época do censo 1996. Enquanto para 1991 foram processados os resultados de 6.343 setores válidos,

Tabela 2

Matriz de fatores para variáveis selecionadas.

\begin{tabular}{lcc}
\hline Variável & Fator 1 & Fator 2 \\
\hline \% dos chefes de domicílio com 15 ou mais anos de estudo & 0,85 & 0,46 \\
Rendimento médio do chefe de domicílio em salário-mínimo & 0,79 & 0,51 \\
Número de banheiros por domicílio & 0,74 & 0,56 \\
\% da população com 65 ou mais anos de idade & 0,72 & $-0,45$ \\
\% de domićlios com coleta domiciliar de lixo doméstico feita & 0,64 & $-0,53$ \\
por serviço sem a interveniência do morador & 0,54 & $-0,55$ \\
\% de domicílios com abastecimento de água com canalização interna & 0,53 & $-0,41$
\end{tabular}

* Em valor absoluto. 
com o procedimento adotado - pareamento de unidades censitárias -, reduziu-se o número de setores válidos em aproximadamente 5\% (6.005 válidos para análise).

A investigação foi orientada pela variação dos IQS91 e IQS91*, o que nos permite especular sobre possíveis alterações no perfil de desigualdades a partir da classificação dos setores censitários em três categorias: melhor, pior e igual (Tabela 3).

Deve-se registrar que, com esse recurso podem-se avançar hipóteses preliminares sobre a qualidade dos dados dos censos 1991 e 1996, sobre a estabilidade do índice em apreciação e como o valor da escolaridade irá interferir na classificação por clusters de 1991. Admitindose que as variáveis referentes à infra-estrutura, em termos de percentuais de cobertura do serviço, da mesma forma que a variável demográfica, pouco se alteraram no intervalo de cinco anos, viabiliza-se uma forma plausível de comparação para o meio da década de 1990. Não se deve perder de vista que há limitações nesta solução e hipóteses mais estruturadas terão de aguardar pelas análises do censo 2000, mas após os resultados o leitor poderá também tirar suas próprias conclusões.

\section{Resultados}

Procuraremos com o artifício metodológico implementado, responder às seguintes perguntas: (1) qual o efeito da substituição do valor da variável 1996 em 1991 na distribuição?; (2) segundo o partido analítico adotado, que indicações se podem fazer sobre as mudanças detectadas: quem realizou os ganhos e onde se localizaram?

O primeiro resultado relevante é que, com os valores da escolaridade do chefe de domicílio com 15 ou mais anos de estudo transposta de 1996 para 1991, duas categorias desapareceram, ou seja, não há setores classificados no grupo I (muito alto) nem no VII (muito baixo) (Tabela 4). Esse efeito pode ter sido em conseqüência de um rebaixamento do teto, ou de uma elevação do piso. Com relação à primeira possibilidade, pode-se especular que uma ampliação do número de setores com proporção de chefia de domicílio com alta escolaridade teria sido suficiente para fundir as duas primeiras categorias - "muito alto" com "alto" pelo efeito da ampliação da segunda categoria, o que é reforçado ao se observar a variação percentual positiva, de $123,4 \%$ na categoria 2 (Tabela 4), causada pelo efeito da substituição da variável chefes de domicílio com 15 ou mais anos de estudo. Concomitantemente, é possível

\begin{tabular}{lcc}
\hline Tabela 3 & \\
Escala categórica ordinal de variação do Índice de Qualidade Social (IOS). \\
\hline IOS91* $>$ IOS91 & IOS $91^{*}<$ IOS91 & IOS91* $=$ IOS91 \\
\hline Melhor & Igual \\
\hline
\end{tabular}

Tabela 4

Distribuição de freqüência nos clusters.

\begin{tabular}{lrrrr}
\hline \multirow{2}{*}{ Grupo } & \multicolumn{2}{c}{1991} & \multicolumn{2}{c}{ 1991* $^{*}$} \\
& Freqüência & Válido (\%) & Freqüência & Válido (\%) \\
\hline I & 392 & 6,5 & 0 & 0,0 \\
II & 1.208 & 20,1 & 2.699 & 44,9 \\
III & 379 & 6,3 & 341 & 5,7 \\
IV & 1.843 & 30,7 & 588 & 9,8 \\
V & 1.745 & 29,1 & 2.150 & 35,8 \\
VI & 346 & 5,8 & 227 & 3,8 \\
VII & 92 & 1,5 & 0 & 0,0 \\
Total & 6.005 & 100,0 & 6.005 & 100,0 \\
\hline
\end{tabular}

que tenha ocorrido um efeito bastante comum neste tipo de classificação, o efeito umbral: como todos os chefes de domicílio que ultrapassam a barreira dos 14 anos de escolaridade passam a ser classificados na última faixa (15 anos ou mais), a mesma vai perdendo capacidade discriminatória.

No que tange à categoria "muito baixo", pode-se cogitar que a ausência desse cluster deve-se ao fato de que na análise original (apenas as variáveis de 1991), o número de setores censitários classificados nessa categoria foi pequeno, sendo eles, por suas características de grande precariedade e vulnerabilidade social, sensíveis a qualquer alteração. Examinese a Tabela 5 .

Observe-se que, na faixa entre 1 a 7 anos de estudo, houve diminuição no número de chefes de domicílio entre 7 e $8 \%$. A faixa de 8 a 10 anos apresentou crescimento tênue, registrando-se nas duas faixas superiores aumentos da ordem de $10 \%$. Houve um deslocamento aproximadamente simétrico entre as duas faixas de escolaridade inferiores e as duas superiores. Observe-se que se os indivíduos que integravam o conjunto que possuía 3 anos de estudo em 1991, conseguiram acrescentar cinco anos à sua formação, passaram da faixa de um a três para a de 8 a 10, que assinala o primeiro grau 
completo. O mesmo pode-se supor para o conjunto de indivíduos que estava com sete anos de estudo, tendo passado para a faixa 11 a 14 anos, que assinala o segundo grau completo. Apoiados em estudos da Pesquisa Nacional por Amostra de Domicílio (PNAD), sabe-se que a faixa de 8 a 10 anos representa um limiar importante (Bastos, 2001; Scalon, 1999).

Podem-se sintetizar as conclusões, até esse ponto, da seguinte forma: houve uma compressão na distribuição relativamente aos clusters, ou categorias. O desaparecimento da categoria, ou cluster, "muito alto" deveu-se a um efeito umbral, e não ao rebaixamento do teto da distribuição; com relação à categoria, ou cluster, "muito baixo", a supressão deveuse, isso sim, a uma elevação do piso através de um deslocamento simétrico entre as faixas inferiores e superiores de escolaridade: pode-se cogitar que ocorreu um deslocamento geral para cima, pela elevação do patamar, tomando-se por referência a variável chefes de domicílio com 15 ou mais anos de estudo. Especulase, a partir de análises das PNADs, de que há indícios que a faixa 8 a 10 anos de estudo fun-

Tabela 5

Variação percentual dos anos de estudo do chefe de domicílio.

Município do Rio de Janeiro, Brasil, 1991 e 1996.

\begin{tabular}{lccc}
\hline $\begin{array}{l}\text { Anos de estudo do chefe } \\
\text { de domicílio }\end{array}$ & $1991(\%)$ & $1996(\%)$ & Variação (\%) \\
\hline $1-3$ & 9,9 & 9,1 & $-8,0$ \\
$4-7$ & 28,5 & 26,5 & $-7,0$ \\
$8-10$ & 15,8 & 16,1 & 2,0 \\
$11-14$ & 21,2 & 23,4 & 11,0 \\
15 ou + & 16,7 & 18,4 & 10,0 \\
\hline
\end{tabular}

Tabela 6

Distribuição dos setores segundo a variação do Índice de Qualidade Social (IQS).

\begin{tabular}{lrc}
\hline IQS91-IOS91* & Número de Setores & $\%$ \\
\hline Melhor & 1.834 & 30,5 \\
Pior & 867 & 14,4 \\
lgual & 3.304 & 55,0 \\
Total & 6.005 & - \\
\hline
\end{tabular}

ciona com um limiar difícil de ser transposto, o que poderia explicar sua variação percentual pífia.

Todas as hipóteses apresentadas até o momento referem-se ao conjunto. No sentido de buscar extrair mais informações dos dados, pode-se refinar um pouco mais a análise gerando-se outra classificação, baseada na mudança do cluster em que o setor censitário foi classificado, ou seja, seu valor com os dados originais de 1991 e seu valor com os valores de 1996 em 1991 , segundo as categorias apresentadas na Tabela 3, seguida da análise da variância, testando-se a hipótese de que as médias dos três grupos são iguais $\left(\mathrm{H}_{0}\right)$. Os resultados estão sintetizados nas Tabelas 6 e 7 .

A ANOVA indica que a hipótese de que os grupos possuem médias iguais deve ser rejeitada. Pode-se afirmar, com 95\% de certeza, que os três conjuntos de setores censitários agrupados pela comparação entre os resultados de 1991 e o efeito da alteração do valor da variável, assumindo os valores de 1996, formam três conjuntos distintos. O teste de homogeneidade confirma a existência de três grupos, e indica que quem melhorou foi quem possuía as médias mais baixas para as variáveis de escolaridade alta, tanto em 1991 como em 1996 (Tabela 8).

Em suma, se o valor da escolaridade alta em 1991 fosse o de 1996, a análise multivariada (componentes principais e clusters) teria resultado numa distribuição mais homogênea e os seus maiores efeitos - mudanças de classificação para categorias de melhor conteúdo social - teriam sido sentidos em setores censitários com menor percentual de chefes de domicílio com escolaridade elevada, sugerindo que a melhora se fez na direção de uma maior homogeneidade ou de uma diminuição das desigualdades. Pode-se especular, portanto, a favor de efeitos redistributivos. Pode-se argumentar que o efeito umbral pode responder por esse fenômeno, o que procede, mas aqui estamos interessados em resultados preliminares, uma vez que apenas com os dados do censo 2000 poderão ser dirimidas as dúvidas.

Com as variáveis de que se dispõe para aprofundar a investigação, pode-se verificar, apenas para os dados de 1991, que a melhora ocorreu em áreas em que a proporção de mulheres chefes de domicílio tendia a ser maior. É o que se pode observar na Tabela 9: no grupo que teve elevação (ou não sofreu alteração) na classificação, segundo o IQS, a participação percentual das mulheres, como chefes de domicílio, era mais elevada, ou seja, a melhora pode ser devida à elevação da escolaridade entre as 


\begin{tabular}{|c|c|c|c|c|c|}
\hline Variáveis & $\begin{array}{l}\text { Soma dos } \\
\text { quadrados }\end{array}$ & $\begin{array}{l}\text { Graus de } \\
\text { liberdade }\end{array}$ & $\begin{array}{l}\text { Média dos } \\
\text { quadrados }\end{array}$ & $\mathrm{F}$ & Significância \\
\hline \multicolumn{6}{|c|}{$\begin{array}{l}\text { Escolaridade do chefe de domicílio com } \\
15 \text { ou mais anos de estudo, } 1991\end{array}$} \\
\hline Entre os grupos & $299.768,395$ & 2 & $149.884,198$ & 501,150 & 0,000 \\
\hline Dentro dos grupos & $1.795 .079,915$ & 6.002 & 299,080 & & \\
\hline Total & $2.094 .848,310$ & 6.004 & & & \\
\hline \multicolumn{6}{|c|}{$\begin{array}{l}\text { Escolaridade do chefe de domicílio com } \\
15 \text { ou mais anos de estudo, } 1996\end{array}$} \\
\hline Entre os grupos & $275.476,753$ & 2 & $137.738,377$ & 387,046 & 0,000 \\
\hline Dentro dos grupos & $2.135 .936,768$ & 6.002 & 355,871 & & \\
\hline Total & $2.411 .413,521$ & 6.004 & & & \\
\hline \multicolumn{6}{|l|}{ Fator 1} \\
\hline Entre os grupos & 624,027 & 2 & 312,014 & 348,088 & 0,000 \\
\hline Dentro dos grupos & $5.379,973$ & 6.002 & 0,896 & & \\
\hline Total & $6.004,000$ & 6.004 & & & \\
\hline
\end{tabular}

mulheres ou ser mais intensa entre os grupos sociais com baixo IQS.

Argumentos a favor dessa possibilidade ganham apoio ao se observarem os gráficos apresentados nas figuras a seguir: nos dois primeiros apresenta-se a relação entre os percentuais de chefes de domicílio com 15 ou mais anos de estudo e mulheres chefes de domicílio, para 1991 e 1996, respectivamente - (Figura 1, Figura 2). A reta ajustada indica uma correlação negativa, com coeficientes de Pearson próximos $(-0,33 /-0,35)$.

Quando o mesmo diagrama de dispersão é traçado para uma área selecionada da cidade onde a classificação foi alterada para melhor (Ilha do Governador - Figura 3), a relação se inverte, passando a indicar uma correlação positiva com coeficiente de Pearson da ordem de 0,56 , entre ser chefe de domicílio possuindo escolaridade elevada (15 ou mais anos) e ser o chefe de domicílio mulher. O padrão se repete e fortalece o argumento, quando traçamos o diagrama de dispersão para todos os setores que tiveram a classificação alterada para melhor com o recurso metodológico especificado (Figura 4).

A síntese dos resultados é a seguinte: houve discreto crescimento da escolaridade nas faixas superiores dos anos de estudo. O efeito da variável chefes de domicílio com 15 ou mais anos de estudo de 1996 em 1991, é um achatamento na distribuição das categorias dos clus-
Tabela 8

Médias por categorias segundo escolaridade superior (15 ou mais anos de estudo) para os chefes de domicílio.

\begin{tabular}{lrcc}
\hline IQS91/IOS91* & 1991 & 1996 & Variação (\%) \\
\hline Melhor & 9,9 & 11,7 & 18,3 \\
Igual & 16,4 & 18,1 & 10,8 \\
Pior & 32,4 & 33,3 & 2,7 \\
Total & 16,7 & 18,4 & 9,9 \\
\hline
\end{tabular}

Tabela 9

Variação percentual dos anos de escolaridade dos chefes de domicílio e proporção de mulheres chefes de domicílio, 1991/1996.

\begin{tabular}{lcccccc}
\hline IOS91/IOS91* & \multicolumn{7}{c}{ Variação (anos) } \\
& $1-3$ & $4-7$ & $8-10$ & $11-14$ & 15 e + & $\begin{array}{l}\text { \% de mulher } \\
\text { chefe de domicílio } \\
\text { entre os chefes }\end{array}$ \\
\hline Melhorou & 12,2 & $-3,2$ & 13,4 & 45,4 & 84,2 & 27,1 \\
Igual & 13,4 & $-4,9$ & 7,9 & 32,0 & 108,8 & 27,6 \\
Piorou & 37,9 & 0,02 & 4,6 & 12,6 & 13,2 & 23,7 \\
\hline
\end{tabular}


Figura 1

Diagrama de dispersão entre mulheres chefes de domicílio (PP_MUCHE) e chefes de domicílio com 15 ou mais anos de estudo (P_ES15M), 1991.

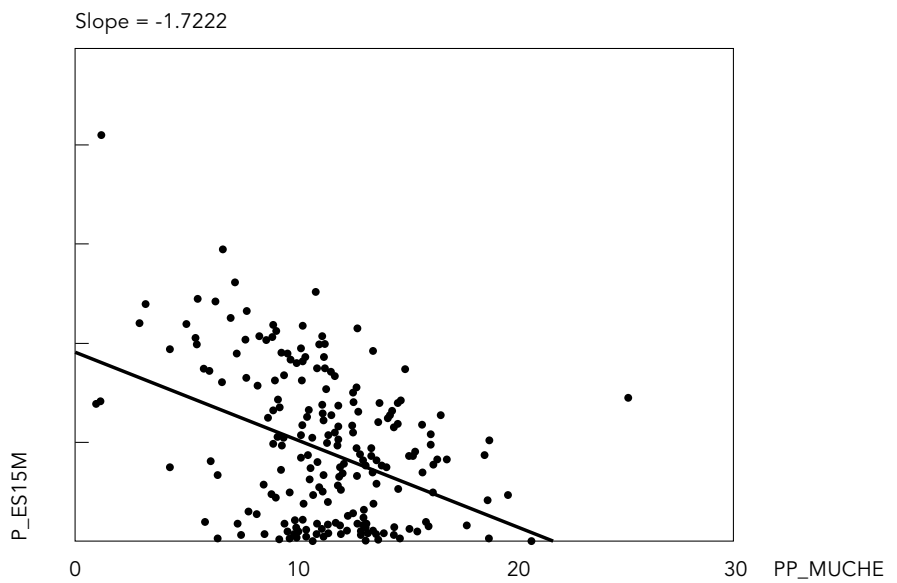

Figura 2

Diagrama de dispersão entre mulheres chefes de domicílio (PP_MUCHE) e chefes de domicílio com 15 ou mais anos de estudo (P_ES15M96), 1996.

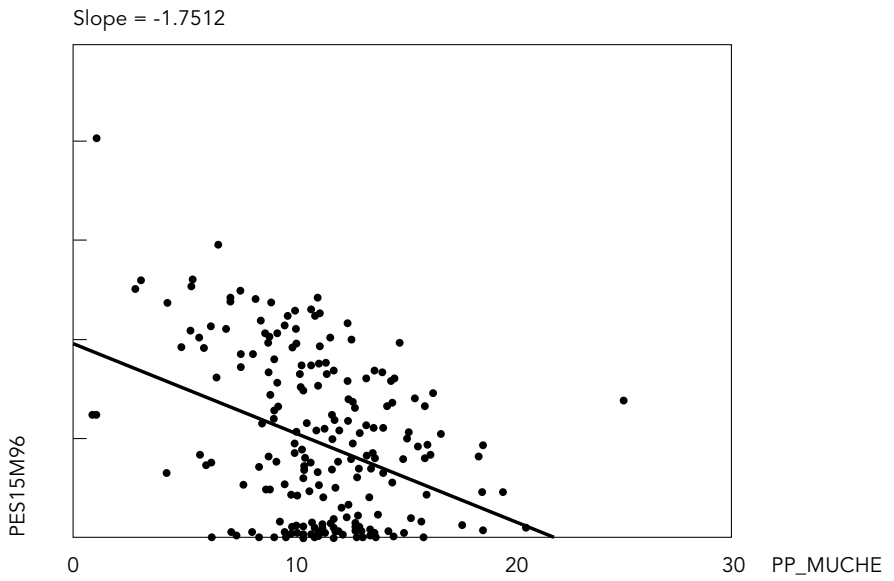

ters, com o desaparecimento das classes extremas, sugerindo ter havido um efeito umbral, repercutindo na classificação dos clusters. A maioria dos setores continuou na mesma categoria, mas os que tiveram sua classificação alterada por efeito da variável em 1996, apresentam-se com o maior percentual de mulheres chefes de domicílio em 1991, sugerindo esse grupo como o principal responsável pelo aumento dos níveis de escolaridade no Município do Rio de Janeiro. Resta agora investigar a localização dos setores que passaram para um cluster de maior status social.

Conforme pode ser lido na Tabela 10, nas regiões administrativas de Jacarepaguá, Irajá, Bangu, Méier, Madureira e Penha, encontra-se o maior número de setores $(58,5 \%)$ no qual o efeito da variável escolaridade alta de $1996 \mathrm{fez}$ com que os mesmos fossem classificados em um cluster mais alto, nesse sentido teriam melhorado.

Como conclusão dos resultados, foi calculado um índice de escolarização para os dois anos, 1991 e 1996, a partir da seguinte fórmula:

$$
i c_{x}=\frac{\sum_{i}^{n} m_{i} p_{i}}{\sum p_{i}}
$$

onde $i c_{x}$ é o índice de escolarização para o ano $x, m_{i}$ o ponto médio em cada faixa de escolaridade $i$ e $p_{i}$ a população por faixa de escolaridade.

Os resultados são: 9,1 e 9,7 anos de escolaridade para 1991 e 1996, respectivamente, o que significa um acréscimo de aproximadamente $6 \%$ em cinco anos. Ou, em outras palavras, que os chefes de domicílio, na área relatada, acrescentaram anos de escolaridade à sua formação na razão de 0,12 anos de escolaridade por ano.

\section{Discussão}

O efeito causado pela alteração do valor de uma variável de escolaridade alta apenas vem confirmar a sua importância específica, de resto fartamente confirmada pela literatura (Pastore \& Silva, 1999). Se o valor da escolaridade em 1991 fosse o de 1996, para os setores válidos, poderia se cogitar sobre um ganho geral de escolaridade e o indício de diminuição deste tipo de desigualdade. A queda geral da taxa de fecundidade e o aumento da escolaridade feminina poderiam ser suficientes para apoiar essas indicações, que deverão ser investigadas com mais detalhe para o censo 2000.

Estudos sobre o aumento do acesso à educação, e conseqüentes melhores oportunida- 
des sociais têm proporcionado dados consistentes que apontam maior número de anos de estudo como fator diminuidor da possibilidade de ser pobre, além de ser o principal responsável por maiores oportunidades de ascensão sociocultural. Estudo produzido por Heringer (2002), compara a situação educacional no Brasil em 1988 e em 1996, verificando que houve um aumento da escolaridade dos brasileiros nesse período. Em 1988, 4,9\% da população brasileira tinha 12 anos ou mais de estudo. Essa proporção aumentou para 7,5\% em 1996, utilizando-se dos dados das PNADs de 1988 e 1996 (IBGE, 1988, 1996).

Neri \& Soares (2002) apontam que o número médio de anos completos de estudo varia bastante entre chefes pobres e não-pobres, e que este dado corrobora a hipótese de que anos completos de estudo são a aproximação mais razoável para renda permanente encontrada nas pesquisas de domicílio. Para se ter uma idéia de valores, os não-pobres têm cerca de dois anos a mais de estudo do que os pobres. Parece mesmo que a pobreza no Brasil diminuiu entre 1991 e 1996, período no qual houve mudanças significativas no plano social, graças aos efeitos do plano de estabilização (Plano Real). Rocha (2000), fez uma análise das últimas décadas e concluiu que quaisquer que sejam os valores das linhas de pobrezas que se utilizem, temos uma diminuição da pobreza entre as décadas de 70 e 80 . A partir deste momento, seguem-se flutuações até 1993 e, em 1994, a incidência de pobreza diminui consideravelmente devido ao plano de estabilização, mantendo-se praticamente inalterada deste então.

Na relação de anos de estudo versus pobreza, verifica-se que, de todas as variáveis disponíveis, a de baixa escolaridade (menos de quatro anos de estudo), é a que mais se relaciona com a probabilidade de um indivíduo ser pobre, e nesse sentido, a variável educação é ainda mais importante do que, por exemplo, as variáveis sexo e cor, também muito exploradas nos estudos sobre desigualdade social (S. Rocha, 2001, em apresentação feita durante o seminário Saúde e Desigualdade: Instituições e Políticas Públicas no Século XXI). Aspecto também registrado no trabalho de Rocha é o de que estudos com base em dados da década de 1980 demonstram que, no Brasil, cada ano de estudo a mais significa aumento de renda entre $10 \%$ e $19 \%$.

Cabe mencionar que uma diminuição na desigualdade social está fortemente correlacionada a uma melhor situação de saúde. Estudo de Szwarcwald et al. (1999:22), testando a asso-
Figura 3

Diagrama de dispersão para os setores censitários da llha do Governador com classificação alterada para melhor entre 1991 e 1996.

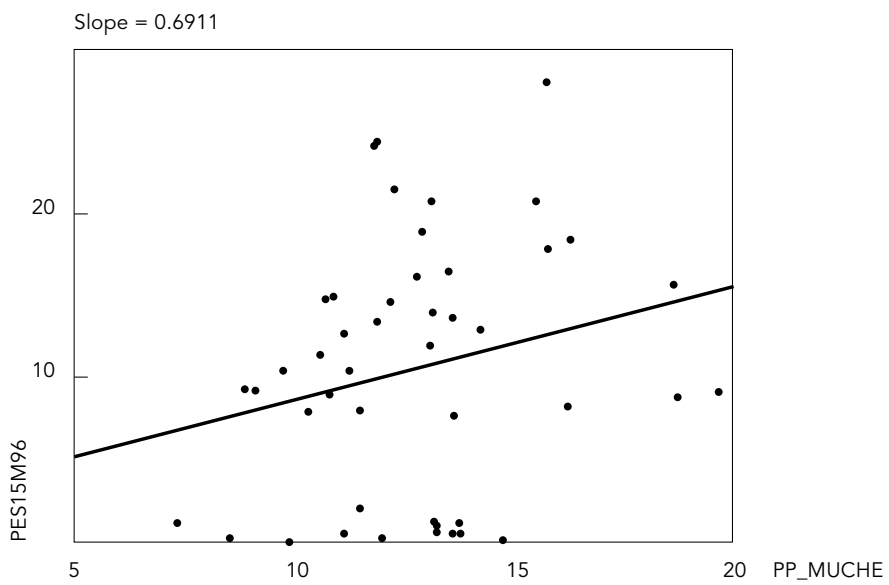

Figura 4

Diagrama de dispersão para todos os setores censitários com classificação alterada para melhor entre 1991 e 1996.

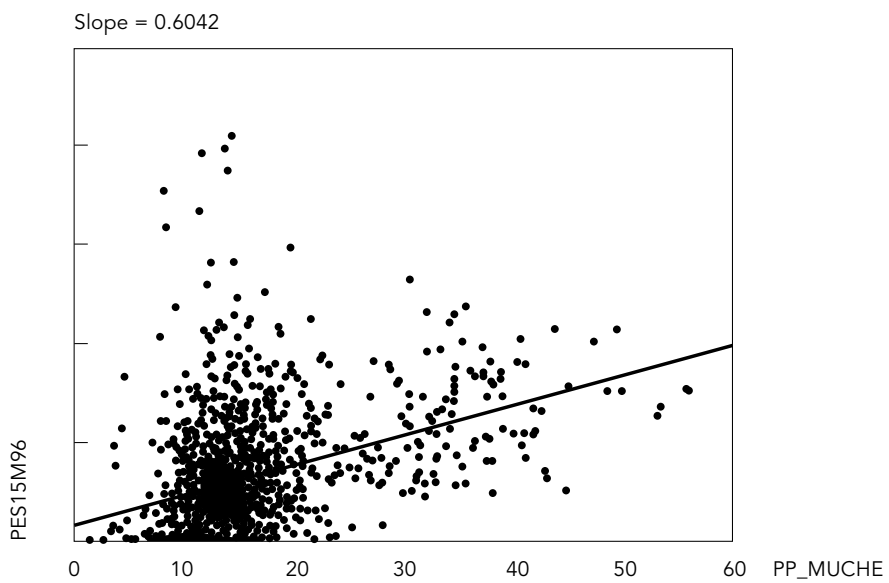


ciação entre desigualdade de renda e condições de saúde no Município do Rio de Janeiro, afirma que "todos os indicadores de concentração de renda (...), mostraram-se correlacionados entre si e significativamente correlacionados aos indicadores de saúde". Além da própria questão econômica que influencia o acesso a serviços médicos, existe uma relação também no fato de que pais com maior grau de educação são mais eficientes no cuidado com seus filhos, utilizando melhor os recursos de saúde existentes (tanto próprios como os proporcionados pelo estado) (Musgrove, 1984).

Dentro dessa linha investigativa, o trabalho realizado por Silva \& Hasenbalg (2002) é bastante elucidativo para verificar as transições educacionais no Brasil (da primeira à oitava série). A partir de um modelo logístico para análise da progressão escolar, distinguiram fatores que influenciam as transições escolares, nível a nível, permitindo que se conheçam, para cada transição, os principais fatores de impedimento para a ultrapassagem de cada um dos patamares (séries). Como resultado, encontraram que a maior parte dos determinantes sócioeconômicos, tais como as variáveis de região e área de residência, ligadas à estrutura familiar e gênero, têm o seu impacto máximo no meio do ciclo escolar básico, sendo mais fracos tanto no início como, sobretudo, depois destas transições centrais. Já a variável escolaridade do chefe de família é a única que se atenua conforme se move para transições superiores, contrariando a expectativa do estudo de que todas as variáveis viessem a se comportar dessa maneira.

Resta assinalar que vários estudos têm demonstrado uma redução nos níveis de pobreza a partir das transformações, em decorrência do ajustamento da economia brasileira ao contexto global, especialmente a partir de 1994. Barros (2000), ao analisar a situação brasileira no que tange à pobreza e à desigualdade nas décadas de 1980 e 1990, identifica dois momentos em que se observa redução no nível de pobreza da população. O primeiro está diretamente relacionado à implantação do Plano Cruzado, sendo seu impacto bastante expressivo, com o percentual de pobres caindo de $43,6 \%$ para $28,2 \%$ da população. Seu efeito, entretanto, foi efêmero, pois já no ano seguinte, registrava-se uma aproximação do percentual ao patamar anterior, com $40,9 \%$ em 1987 . O segundo momento refere-se ao conjunto de medidas associadas ao ajustamento de 1994, com características bastante distintas da redução anteriormente observada. Comparativamente ao ajustamento de 1986, se por um lado, a que- da no percentual de pobres foi bem mais modesta, caindo de um patamar de 41,7\% em 1993 para $33,9 \%$ em 1995, por outro lado, o novo patamar alcançado manteve-se estável até o final da década de 1990. Vale destacar que a redução do número de pobres não significou diminuição das desigualdades de renda, tendo o coeficiente de Gini permanecido em torno de 0,60 no período 1993-1999.

Embora não se possam estabelecer ligações diretas e imediatas, pode-se supor, com base no que foi apresentado anteriormente, que os resultados aqui discutidos sejam devidos aos ganhos realizados pela população mais pobre, a partir das grandes transformações dos últimos 15 anos. Ou seja, o efeito da substituição do valor da variável em 1996 na distribuição de 1991 alterou o IQS, que relata os efeitos dessas transformações. Deve-se lembrar, mais uma vez, que a associação entre escolaridade e renda é bastante clara no Brasil. Especificamente para o âmbito dos dados analisados é alta: 0,90. Essa associação pode ser visualmente constatada na Figura 5, onde estão plotados a proporção de chefes de domicílio com 15 ou mais anos de estudo contra o rendimento dos mesmos chefes para o Município do Rio de Janeiro.

Note-se ainda, o rompimento da linearidade na parte alta da distribuição (assinalado na Figura 5), evidenciando um deslocamento entre anos de escolaridade e rendimentos do chefe do domicílio a favor dos rendimentos. Conforme já registrado (Tabela 6), aqueles que tiveram sua classificação alterada para melhor formam um grupo cujo valor médio da variável escolaridade alta (15 ou mais anos de estudo) é menor que nos outros dois grupos. Este grupo é também aquele que apresenta a menor média para a variável "renda do chefe em 1991", reforçando a suposição de que os efeitos dos ajustes estruturais podem estar sendo captados pelos resultados do IQS91*.

Para concluir e a título de maior qualificação dos resultados aqui discutidos, considerese o seguinte: no início da década passada existiam aproximadamente 550 favelas no Município do Rio de Janeiro. Essas áreas concentravam $17,6 \%$ da população carioca no ano de 1991. Em relação ao IQS91, embora $58,6 \%$ do setores censitários classificados como favela tenham se concentrado no cluster V (IQS Baixo), é importante destacar que esse tipo de setor compõe 75,5\% do cluster VI (IQS Baixo/Muito Baixo) e $85,9 \%$ do VII (IQS Muito Baixo). Isso significa dizer que o efeito de elevação do piso que leva ao desaparecimento do cluster VII no IQS91*, por si só já demonstra ter um significativo impacto sobre áreas de favela. 
A Figura 6 chama a atenção para a heterogeneidade na composição social dos espaços classificados como favela. Isso demonstra que a oposição favela/não-favela é apropriada a alguns níveis de análise, mas não a outros. Esses espaços não são homogêneos em relação à pobreza e às condições de vida e, muitas vezes tendem a apresentar, sob certos aspectos, mais similaridades que diferenças em relação a outras áreas da chamada cidade formal. Preteceille \& Valladares (2000) e Valladares (2001), em apresentação feita durante o Fórum América Latina Habitar 2000, também detectaram em seus estudos essas mesmas características de heterogeneidade nas favelas cariocas e assinalaram a importância dessa constatação para o planejamento da focalização das políticas sociais. Ou seja, a visão da favela como território exclusivo da pobreza desvia o olhar de outras áreas tão ou mais carentes, além de alimentar o preconceito em relação aos espaços e pessoas rotulados com os títulos de favelas ou favelados. Cientes das limitações da dicotomia favela/não-favela, considera-se que, para o tipo de análise que se empreende, a comparação entre os dois grupos justifica-se, e é capaz de oferecer boas pistas sobre o que teria acontecido nessas áreas no período 19911996.

Para saber em que medida os setores "favela" situados nos demais clusters também se beneficiaram dos efeitos positivos decorrentes do controle para a variável escolaridade alta em 1996, comparou-se a variação do IQS (melhor- igual-pior) nos grupos favela e não-favela, testando a hipótese de que o pertencimento a um ou outro grupo inclina a padrões diferenciados na variação do IQS. O resultado do teste de qui-quadrado permite-nos afirmar que a hipótese é verdadeira, e que o grupo favela, proporcionalmente, apresenta maiores ganhos de qualidade. O teste de comparação de médias (ANOVA) também permite-nos afirmar que a variação da proporção de chefes com escolari-

Figura 5

Diagrama de dispersão entre a proporção de chefes de domicílio com 15 ou mais anos de estudo (PES15M91), contra o rendimento médio nominal em salários mínimos dos mesmos chefes (SM_REMCH), 1991.

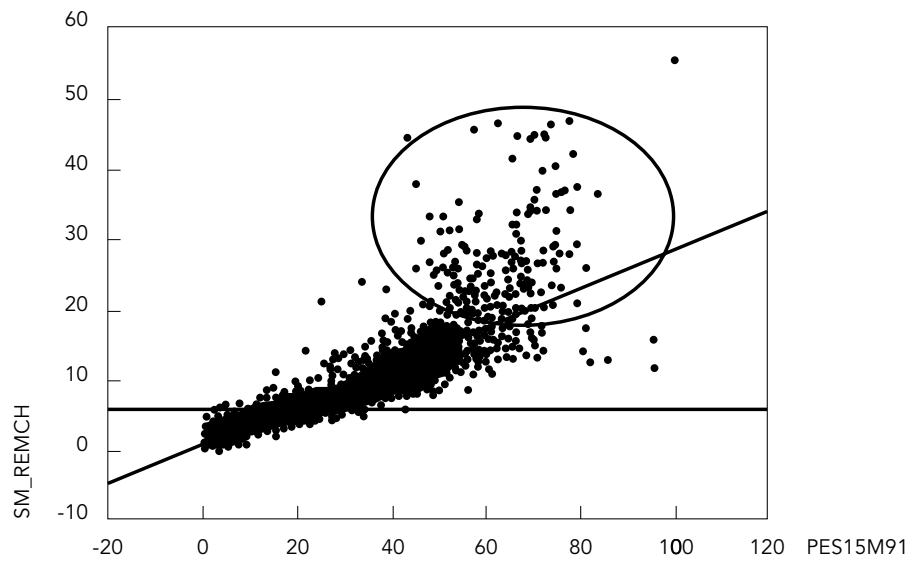

Figura 6

Proporção de setores censitários classificados como favela/não-favela no interior dos clusters.

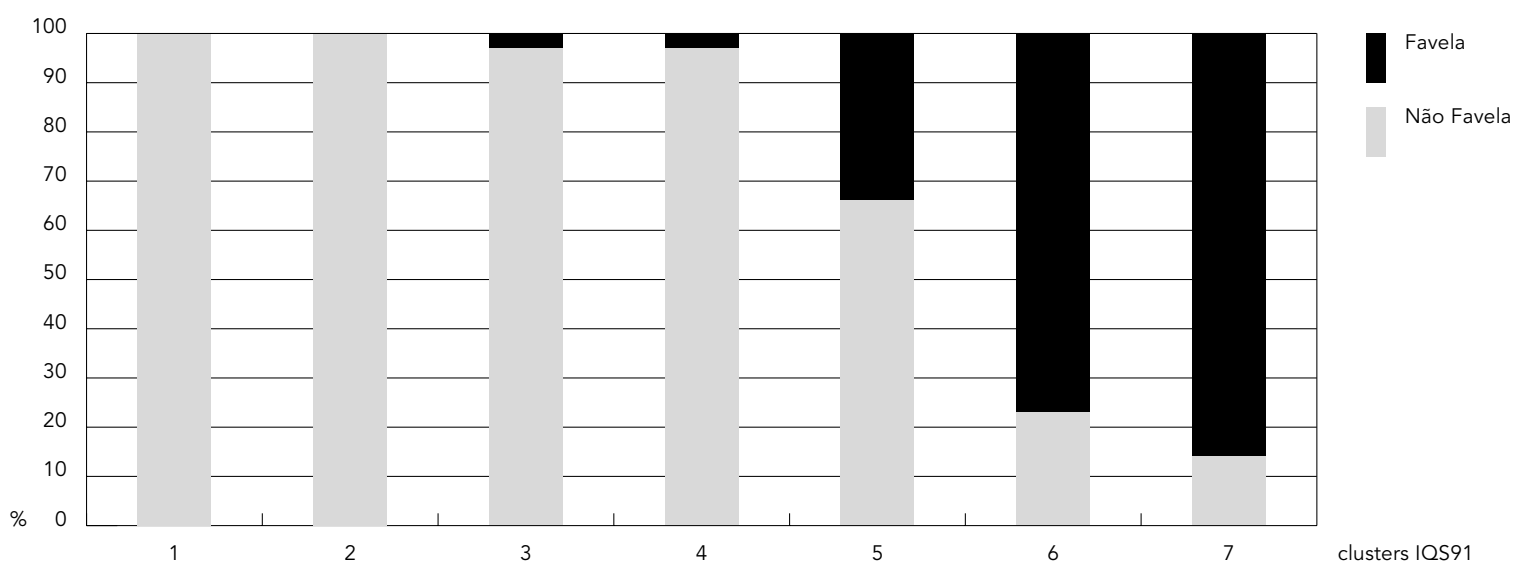


Tabela 10

Distribuição de freqüência dos setores com Índice de Qualidade Social (IQS)

alterado para melhor pelo efeito de 1996, por região administrativa.

Município do Rio de Janeiro, Brasil.

\begin{tabular}{|c|c|c|c|}
\hline Região Administrativa & Freqüências & $\%$ & $\%$ acumulado \\
\hline Portuária & 20 & 1,1 & 1,1 \\
\hline Guaratiba & 24 & 1,3 & 2,4 \\
\hline Lagoa & 25 & 1,4 & 3,8 \\
\hline Tijuca & 26 & 1,4 & 5,2 \\
\hline Barra da Tijuca & 27 & 1,5 & 6,7 \\
\hline Pavuna & 28 & 1,5 & 8,2 \\
\hline Rio Comprido & 31 & 1,7 & 9,9 \\
\hline Copacabana & 38 & 2,1 & 11,9 \\
\hline Santa Cruz & 39 & 2,1 & 14,1 \\
\hline Centro & 40 & 2,2 & 16,2 \\
\hline Anchieta & 40 & 2,2 & 18,4 \\
\hline Santa Teresa & 40 & 2,2 & 20,6 \\
\hline Vila Isabel & 46 & 2,5 & 23,1 \\
\hline Ilha do Governador & 46 & 2,5 & 25,6 \\
\hline São Cristóvão & 46 & 2,5 & 28,1 \\
\hline Campo Grande & 52 & 2,8 & 31,0 \\
\hline Botafogo & 55 & 3,0 & 34,0 \\
\hline Ramos & 68 & 3,7 & 37,7 \\
\hline Inhaúma & 70 & 3,8 & 41,5 \\
\hline Jacarepaguá & 116 & 6,3 & 47,8 \\
\hline Irajá & 119 & 6,5 & 54,3 \\
\hline Bangu & 136 & 7,4 & 61,7 \\
\hline Penha & 172 & 9,4 & 71,1 \\
\hline Méier & 261 & 14,2 & 85,3 \\
\hline Madureira & 269 & 14,7 & 100,0 \\
\hline Total & 1.834 & 100,0 & \\
\hline
\end{tabular}

dade alta é significativamente maior nesse grupo (Tabelas 11 e 12).

\section{Conclusão}

O presente artigo realizou um exercício empírico e metodológico com o objetivo de explorar analiticamente as transformações sofridas pela distribuição da estrutura social no espaço carioca durante a década de 1990. Para além dos resultados concretos, o trabalho apresenta um inventivo caminho metodológico para o estudo da dinâmica social na cidade, potencialmente utilizável para outras realidades urbanas e outros períodos.

Partindo da classificação de setores censitários segundo seus conteúdos sociais construída em Najar (1997), realizamos a substituição, no índice sintético criado por aquele trabalho, da variável escolaridade do chefe do domicílio do censo de 1991 pela mesma variável obtida pela contagem populacional de 1996. Com o índice resultante desse exercício, reprocessamos a classificação dos setores, obtendo resultados que confirmam trabalhos anteriores sobre a dinâmica social dos espaços metropolitanos brasileiros em período recente.

Pudemos observar uma redução da amplitude das situações experimentadas pelos setores, e em especial o desaparecimento das situações muito alta e muito baixa do índice. Com relação à situação social de melhor condição, interpretamos o resultado como causado por efeito metodológico do tamanho da faixa de

Tabela 11

Variação entre 1991 e 1996 para favela e não-favela.

\begin{tabular}{|c|c|c|c|c|c|c|c|c|}
\hline & \multicolumn{2}{|c|}{ Melhor } & \multicolumn{2}{|c|}{ Igual } & \multicolumn{2}{|c|}{ Pior } & \multicolumn{2}{|c|}{ Total } \\
\hline & $n$ & $\%$ & $n$ & $\%$ & $\mathrm{n}$ & $\%$ & $\mathrm{n}$ & $\%$ \\
\hline Não-favela & 1.484 & 29,7 & 2.680 & 53,5 & 841 & 16,8 & 5.005 & 100,0 \\
\hline Favela & 350 & 35,0 & 624 & 62,4 & 26 & 2,6 & 1.000 & 100,0 \\
\hline Total & 1.834 & 30,5 & 3.304 & 55,0 & 867 & 14,4 & 6.005 & 100,0 \\
\hline
\end{tabular}




\begin{tabular}{l} 
Tabela 12 \\
\begin{tabular}{l} 
Variação da proporção de chefes \\
com escolaridade alta. \\
\hline \multicolumn{2}{c}{ Acréscimo de escolaridade } \\
$1991-1996$
\end{tabular} \\
$\begin{array}{l}\text { Não-Favela } \\
\text { Favela } \\
\text { Total }\end{array}$ \\
\hline
\end{tabular}

escolaridade, mas o desaparecimento de setores na situação social de pior condição indica, para nós, uma melhora real nos padrões educacionais. Tudo indica que ocorreu uma melhoria no patamar mínimo de escolaridade da população carioca na primeira metade da década. Os dados mostram que a melhora se concentra principalmente em setores onde a presença de mulheres na chefia das famílias é mais expressiva. Esse resultado se deve aos melho- res padrões educacionais das mulheres, processo já destacado por outros autores, mas pode se dever também, ao menos parcialmente, à maior presença das mulheres como chefes de família em camadas populacionais de mais baixa renda. Efetivamente, essa melhora também se concentra em setores censitários habitados por população de baixa renda (e escolaridade) em 1991 e, em especial, em setores subnormais - definição do IBGE (Fundação Instituto Brasileiro de Geografia e Estatística) para favelas.

Resumindo, as informações indicam uma melhora da situação educacional dos chefes de família no Rio de Janeiro na primeira metade da década de 1990. Essa se concentra em locais onde a presença feminina é mais elevada na chefia das famílias, sugerindo que essa melhora pode ter sido puxada por uma elevação na escolaridade feminina. Além disso, ela se concentra fortemente em grupos sociais de baixa renda, e em especial entre habitantes de núcleos favelados.

\section{Agradecimentos}

Beneficiaram-se grandemente os autores dos comentários e sugestões do Prof. Dr. Sérgio Koifman e do Prof. Dr. Paulo Barata, colegas da Escola Nacional de Saúde Pública, Fundação Oswaldo Cruz, aos quais muito agradecem. Toda e qualquer responsabilidade interpretativa, no entanto, cabe à equipe que desenhou e redigiu o artigo.

\section{Referências}

ABREU, M., 1987. A Evolução Urbana do Rio de Janeiro. Rio de Janeiro: Zahar.

BARROS, R. P., 2000. A estabilidade inaceitável: Desigualdade e pobreza no Brasil. In: Desigualdade e Pobreza no Brasil (R. Henriques, org.), pp. 21-47, Rio de Janeiro: Instituto de Pesquisa Econômica Aplicada.

DUCHIADE, M., 1991. Mortalidade Infantil por Pneumonia na Região Metropolitana do Rio de Janeiro 1976-86. Dissertação de Mestrado, Rio de Janeiro: Escola Nacional de Saúde Pública, Fundação Oswaldo Cruz.

DUnteman, G. H., 1989. Principal Components Analysis. Newbury Park: Sage.

GALVÃO, R. D.; NOBRE, F. F. \& VASCONCELLOS, M. M., 1999. Modelos matemáticos de localização aplicados à organização espacial de unidades de saúde. Revista de Saúde Pública, 33:422-434.

HAMILTON, L. C., 1992. Regression with Graphics: A Second Course in Applied Statistics. Belmont: Duxbury Press.

HERINGER, R., 2002. Desigualdades raciais no Brasil: Síntese de indicadores e desafios no campo das políticas públicas. Cadernos de Saúde Pública, 18(Sup.):57-65. 
IBGE (Fundação Instituto Brasileiro de Geografia e Estatística), 1991. Censo Demográfico 1991. 15 Agosto 2002 <http:/ /www.ibge.net/home/default.php>.

IBGE (Fundação Instituto Brasileiro de Geografia e Estatística), 1996. Contagem da População 1996. 15 Agosto 2002 <http://www.ibge.net/home/ default.php>.

IPEA (Instituto de Pesquisa Econômica Aplicada)/ IBAM (Instituto Brasileiro de Administração Municipal), 1976. Região Metropolitana do Rio de Janeiro: Serviços de Interesse Comum. Brasília: Comissão Nacional de Regiões Metropolitanas e Política Urbana.

JENCKS, C., 1993. Heteropolis. Los Angeles - The Riots and the Strange Beauty of Hetero-Architecture. London: Academy Editions.

JOHNSON, R. A. \& WICHERN, D. W., 1992. Applied multivariate statistical analysis. 3 rd Ed. New Jersey: Prentice-Hall.

LEAL, M. C., 1995. Evolução da Mortalidade Infantil no Estado do Rio de Janeiro na Década de 80: $O$ Componente Neonatal. Tese de Doutorado, Rio de Janeiro: Escola Nacional de Saúde Pública, Fundação Oswaldo Cruz.

MACHADO-DA-SILVA, L. A., 1967. A política na favela. Cadernos Brasileiros, 9:35-47.

MARQUES, E., 1993. Desigualdades Sociais e Infra-estrutura Urbana: A Produção do Saneamento no Rio de Janeiro. Dissertação de Mestrado, Rio de Janeiro: Instituto de Pesquisas e Planejamento Urbano e Regional, Universidade Federal do Rio de Janeiro.

MASSEY, D., 1992. Politics and space/time. New Left Review, 196:65-84.

MUSGROVE, P., 1984. Indicadores de bienestar y salud. Selección y empleo de indicadores socioeconómicos para monitoría y evaluación. Boletín de la Oficina Sanitaria Panamericana, 96:439452.

NAJAR, A. L., 1997. Desigualdades Sociais e Análise Espacial: Uma Aplicação para o Município do Rio de Janeiro Utilizando Sistemas de Informações Geográficas. Tese de Doutorado, Rio de Janeiro: Instituto Universitário de Pesquisas do Estado do Rio de Janeiro.

NAJAR, A. L., 1998. Desigualdades de bem-estar social no município do Rio de Janeiro: Um exemplo de aplicação da ferramenta SIG. In: Saúde e Espaço: Estudos Metodológicos e Técnicas de Análise (A. L. Najar \& E. Marques, org.), pp. 167-197, Rio de Janeiro: Editora Fiocruz.

NAJAR, A. L., 2000. Rio 40 Graus: Metodologia de Seleção de Áreas para Minoração de Desigualdades de Bem-estar e Promoção da Saúde na Região Metropolitana do Rio de Janeiro. Rio de Janeiro: Escola Nacional de Saúde Pública, Fundação Oswaldo Cruz.

NAJAR, A. L. \& MARQUES, E., 1998. Saúde e Espaço: Estudos Metodológicos e Técnicas de Análise. Rio de Janeiro: Editora Fiocruz.

NERI, M. \& SOARES, W., 2002. Desigualdade social e saúde no Brasil. Cadernos de Saúde Pública, 18 (Sup.):77-87.

PASTORE, J. \& SILVA, N., 1999. Mobilidade Social no Brasil. São Paulo: Makron.

PINÇON-CHARLOT, M.; PRETECEILLE, E. \& RENDU,
P., 1986. Ségrégation Urbaine: Classes Sociales et Equipements Collectifs ne Région Parisienne. Paris: Anthropos.

PRETECEILLE, E. \& VALLADARES, L., 2000. A desigualdade entre os pobres - favela, favelas. In: $D e-$ sigualdade e Pobreza no Brasil (R. Henriques, org.), pp. 459-485, Rio de Janeiro: Instituto de Pesquisa Econômica Aplicada.

RIBEIRO, L. C. \& TELLES, E., 2000. Rio de Janeiro: Emerging dualization in a historically unequal city. In: Globalizing Cities: A New Spatial Order? (P. Maruse \& R. Kempen, ed.), pp. 78-94, London: Blackwell.

ROCHA, S., 2000. Estimação de linhas de indigência e da pobreza: Opções metodológicas no Brasil. In: Desigualdades e Pobreza no Brasil (R. Henriques, org.), pp. 109-127, Rio de Janeiro: Instituto de Pesquisa Econômica Aplicada.

RUMMEL, R. J., 1970. Applied Factor Analysis. Evanston: Northwestern University Press.

SANTOS, C., 1980. Velhas novidades nos modos de urbanização brasileiros. In: Habitação em Questão (L. Valladares, org.), pp. 17-47, Rio de Janeiro: Zahar.

SANTOS, C. \& BRONSTEIN, O., 1978. Metaurbanização: O caso do Rio de Janeiro. Revista de Administração Municipal, 25:6-34.

SCALON, M. C., 1999. Mobilidade Social no Brasil: Padrões e Tendências. Rio de Janeiro: Editora Revan/Instituto Universitário de Pesquisas do Rio de Janeiro/Universidade Cândido Mendes.

SILVA, N. \& HASENBALG, C., 2002. Recursos familiares e transições educacionais. Cadernos de Saúde Pública, 18(Sup.):67-76.

SZWARCWALD, C.; BASTOS, F. I.; ESTEVES, M. A. P.; ANDRADE, C. L. T.; PAEZ, M. S.; MEDICI, E. V. \& DERRICO, M., 1999. Desigualdade de renda e situação de saúde: O caso do Rio de Janeiro. Cadernos de Saúde Pública, 15:15-28.

TORRES, H. \& MARQUES, E., 2001. Reflexões sobre a hiperperiferia: Novas e velhas faces da pobreza no entorno metropolitano. Revista de Estudos $U r$ banos, 4:49-70.

TIMMS, D., 1971. The Urban Mosaic: Towards a Theory of Residential Differentiation. London: Cambridge University Press.

VASCONCELLOS, M. M., 1997. Modelos de Localização e Sistemas de Informações Geográficas na Assistência Materna e Perinatal: Uma Aplicação no Município do Rio de Janeiro. Tese de Doutorado, Rio de Janeiro: Coordenação dos Programas de Pós-graduação em Engenharia, Universidade Federal do Rio de Janeiro.

VENTURA, Z., 1994. Cidade Partida. São Paulo: Companhia das Letras.

VETTER, D., 1981. A segregação residencial da população economicamente ativa na região metropolitana do Rio de Janeiro, segundo grupos de rendimento mensal. Revista Brasileira de Geografia, 43:587-603.

Recebido em 24 de abril de 2002

Versão final reapresentada em 15 de agosto de 2002 Aprovado em 11 de setembro de 2002 